\title{
Magnetism of Ferromagnetic Metals above their Curie Temperature
}

\author{
P.J. Brown, Grenoble
}

\author{
(Institut Laue-Langevin)
}

Although the $3 \mathrm{~d}$ metals: iron, cobalt and nickel were amongst the first ferromagnetic materials to be recognised, full understanding of their magnetic properties has lagged far behind that of the less widely known magnetic insulators such as europium oxide. The magnetic properties of insulators are well accounted for by the simple Curie Weiss theory in which magnetic moments localised on the magnetic ions respond both to external fields and to an internal field arising from inter-atomic exchange interactions of the Heisenberg type. This theory leads to the well known linear dependence of the inverse magnetic susceptibility on temperature - the Curie Weiss law.

$$
1 / \chi=(T-\theta) / P_{\text {eff }}^{2}
$$

where $\theta$ is a temperature related to the Curie temperature, $P_{\text {eff }}$ is the effective local moment. If the ionic moments are due to electron spin only, as is the case for many transition metal salts, and the total ionic spin is $S$

$P_{\text {eff }}^{2}=4 S(S+1)$ Bohr magnetons ${ }^{2}$ Values of $P_{\text {eff }}$ obtained for transition metal salts lead to integer or half integer values of $S$ which agree with those found in the ordered magnetic state.

Although the paramagnetic susceptibility of many ferromagnetic metals and alloys follows a Curie Weiss law over some range of temperature, the effective moments deduced do not corres-

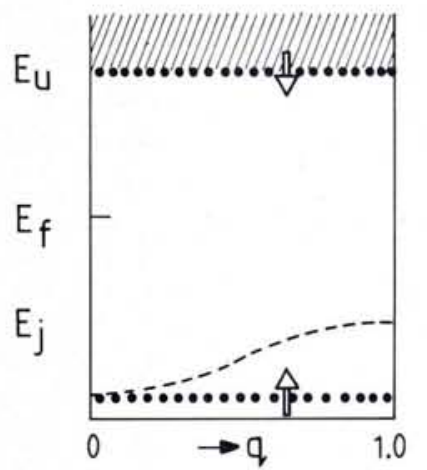

(a) pond to integral or half integral $S$ and are generally not consistent with the moments per atom obtained from the saturation magnetisation. The occurrence of non-integral moments does not pose a problem if it is accepted that the magnetic electrons belong to a partially filled band and thus contribute to the Fermi surface. The saturation magnetisation then measures the mean number of unpaired electrons per atom, and individual atomic moments fluctuate on a time scale of the order $h / W$ where $W$ is the band width.

The first collective electron or band model of ferromagnetism was given by Stoner ${ }^{1}$ ). As in the Curie Weiss theory exchange is introduced as a molecular field proportional to the mean magnetisation. This exchange field produces an energy difference between the spin-up and spin-down electron bands causing electrons to move from one band to the other to maintain a constant electrostatic potential. The imbalance in the population of the bands gives rise to the magnetisation. Stoner showed that, with a positive exchange parameter greater than a certain critical value the magnetisation-induced band splitting would be self sustaining below a critical temperature, giving rise to a ferromagnetic state. The band structures for the magnetic electrons and the frequency $\omega$ versus wave-vector $q$ spectra of magnetic exci-

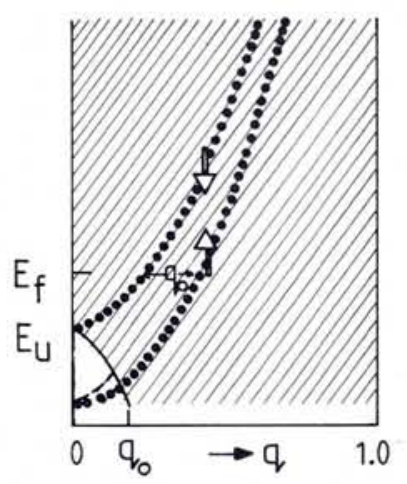

(b)
Fig. 1 - Distribution in q $\omega$ space of electron states (dotted) and magnetic excitations. The dashed lines represent the spin waves, and the hatched areas regions dominated by the Stoner excitations. The Heisenberg and Stoner limits are represented in (a) and (b) respectively. $E_{f}$ is the Fermi energy and the wave-vector $q$ is normalised to unity at the Brillouin zone boundary. tations for a local moment (Heisenberg) and a Stoner system are shown schematically in Fig. 1.

For the local moment system, the magnetic electrons are contained in narrow bands separated by an amount $E_{\mathrm{u}}$ which is the intra-atomic exchange, or Hund'srule, energy; one spin band is full and the other empty. The interatomic coupling energy is $E_{i}=\Sigma J_{i} S_{i} \cdot S_{i}<<E_{u}$ being the sum of pairwise interactions between atomic spins $S_{i}, S_{i}$. The magnetic excitations of such a system are collective transverse fluctuations of the atomic moments (spin waves). They have a dispersion for $T \ll T_{c}$ as indicated in Fig. 1a with the energy of the spin-wave at the Brillouin zone-boundary being $\cong$ $k T_{c}$. As the temperature is raised towards $T_{c}$ the spin-wave spectrum is thermally populated resulting in a reduction in the ordered component of magnetisation and a consequent lowering (renormalisation) of the spin-wave energies. At the transition temperature, the whole spin-wave spectrum is thermally populated resulting in the complete loss of long-range order. The individual atomic moments point in random directions, but retain the same magnitudes since thermal energies are not sufficient to excite the majority carriers into the minority band.

The Stoner picture of ferromagnetism is illustrated in Fig. 1b. At $T<<T_{\text {c }}$ the majority and minority spin-bands are split by an amount $E_{\mathrm{u}}$ which in this case is only of order $k T$ and less than the band width. Although at $q=0$ a finite energy $E_{u}$ is needed to promote an electron from the majority to the minority band, at $q=q_{0}$ such a transfer can take place without the expenditure of energy. In the Stoner system the magnetic excitations are of two kinds: at low energies and low $q$, collective spin wave excitations can occur as indicated by the dashed curve in Fig. 1b, but over the whole of the hatched area the excitations correspond to transfer of electrons from one band to another (Stoner excitations). These Stoner excitations do not conserve the value of individual atomic moments. In the Stoner system, thermal population of the Stoner excitations which takes place as the temperature 
rises leads to a reduction in the band splitting which goes to zero at the Curie temperature so that in the paramagnetic phase no atomic moments exist.

\section{Itinerant versus Local Moment Models}

One may ask to what extent does either of these extreme models account for the properties of metallic ferromagnets at finite temperatures. Important bulk properties which have to be explained are the magnetic susceptibility, the Curie temperature, the temperature dependence of the atomic volume, and the temperature variation of the specific heat. Analysis of the variation of many properties through the Curie temperature gives strong evidence for the persistence of long-lived magnetic moments in the paramagnetic phase. Important among these phenomena are the susceptibility, the resistivity, and the specific heat. The temperature dependence of the atomic volume and the compressibility of iron and nickel suggest that no dramatic change, which would be consequent on a total loss of moment, occurs at $T_{c}$.

These bulk measurements indicate that the simple Stoner picture cannot be appropriate to ferromagnets such as iron and nickel. On the other hand, whilst the local moment theory can account for many of the bulk properties of metallic magnets it fails to explain satisfactorily the difference in the ordered and effective moments. Non-integer moments as well as spectroscopic studies show that magnetic electrons contribute to the Fermi surface and since the bands are not narrow make the existence of local moments in the paramagnetic phase difficult to understand.

\section{Application of Neutron Scattering Tech- niques}

Neutron scattering is one of the most powerful tools for studying magnetic structure and dynamics at the atomic scale. Neutrons of thermal energies have wavelengths of the same order as interatomic distances and the neutron by virtue of its magnetic dipole moment can exchange energy with, and be scattered by the magnetic fields due to the electrons in a magnetic material. In other words neutron scattering may be used to probe the response of a magnetic system to the small perturbation induced by the magnetic dipole field of the neutron. For quantitative discussion a scattering function $S(q, \omega)$ is defined which is proportional to the scattering cross-section for neutrons scattered with a change of momentum $\hbar q$ and a change in energy $\hbar \omega$. Response theory can then be used to relate the scattering function for magnetic scattering to the imaginary part of the generalised magnetic susceptibility $\left.\chi^{\prime \prime}(q \omega)^{2,3}\right)$

$$
\begin{gathered}
S(\mathbf{q}, \omega)=\left(\hbar / 4 \pi \mu_{\mathrm{B}}^{2}\right)[1-\exp -(h \omega / k T)] \cdot \\
\Sigma_{i=1,2,3}\left(1-\mathbf{q}_{i}^{3} \cdot \chi^{\prime \prime}{ }_{i}(q, \omega)\right.
\end{gathered}
$$

In addition the integral of $S(q, \omega)$ over all $\omega$ is proportional to the Fourier transform of the instantaneous spin-density spindensity correlation function (SDSDCF) which gives a snap-shot picture of the average magnetisation around any atom at a given instant in time.

\section{Magnetic Excitation Below $T$ 。}

Triple axis neutron spectrometry allows the scattering function to be determined over a range of $q$ and $\omega$ limited by practical considerations to be of the same order or less than the incident neutrons' wavelength and frequency. The results obtained for iron and nickel ${ }^{4,5}$ ) are illustrated in Fig. 2 where they are compared with those for the Heisenberg-like ferromagnet EuO. The figure shows the dispersion curve for the acoustic mode of collective magnetic excitations (spin-waves). It can be seen that whereas in EuO excitations propagate at all wave-vectors out to the boundary of the Brillouin zone in accordance with the expectations for a local moment system (Fig. 1a), the results for the metallic magnets is quite different. The figure shows that the 'stiffness' of the spin waves is much greater than for EuO, even allowing for the considerably higher Curie temperatures. The energy of the collective mode rises very sharply with increasing wave-vector and in iron a well defined spin-wave is still apparent at the limit of currently accessible energies $\left.{ }^{6}\right)\left(1.8 k T_{c}\right)$ at which energy it approaches the zone boundary. In nickel on the other hand an abrupt decrease in the spin wave intensity sets in at around $1.1 k T_{c}$ which is thought to be due to the competition with Stoner excitations. In raising the temperature in both iron and nickel, only the energies of spin waves at low $q$ renormalise to zero at $T$, the energies of those with $q \geq 0.25 \AA^{-1}$ decrease only slightly, so that they are still finite at $T_{\mathrm{c}}$.

\section{Neutron Scattering Above $T_{\mathrm{c}}$}

On raising the temperature through $T$ one passes through a region in which the scattering is due to critical fluctuations. In this region there are very large slow long-wavelength fluctuations in the degree of ferromagnetic order, and an associated strong neutron scattering at small $\omega$ and $q$. Above $T_{c^{\prime}}$ in the Heisenberg system one expects residual short-range ferromagnetic order with an inverse correlation range proportional to $\left[T /\left(T-T_{c}\right)\right]^{1 / 2}$ and a paramagnetic scattering function with the double Lorentzian form

$\mathrm{S}(\mathbf{q}, \omega)=M /\left(K^{2}+q^{2}\right) \cdot\left[\Lambda q^{2} /\left(\Lambda^{2} q^{4}+\omega^{2}\right)\right]$ which corresponds to magnetic excitations of a diffusive rather than a propagating type.

There have been a number of different experimental neutron scattering studies of the nature of the magnetic excitations in iron and nickel above $T_{c}$. Those which have perhaps excited the most widespread interest presented evidence for the persistence of propagating spin waves at temperatures well above $T_{c^{\prime}}$ $5,6,7$ ) and have become the object of some controversy. One should point out that although the evidence on magnetic excitations provided by neutron scattering is rather direct, the difficulties in determining the purely magnetic crosssections are formidable because the nuclear scattering is in general considerably stronger than the magnetic scattering.

\section{Neutron Scattering with Polarisation Analysis}

An experimental approach to the problem of separating the magnetic component from other contributions to the neutron scattering is to use a polarised neutron beam - one in which all neutron spins point in a given direction - and to analyse the polarisation of the scattered neutrons. In magnetic scattering as opposed to nuclear scattering, the cross-section depends on the relative orientations of the neutron spins and the wave-vector of the momentum transfer.

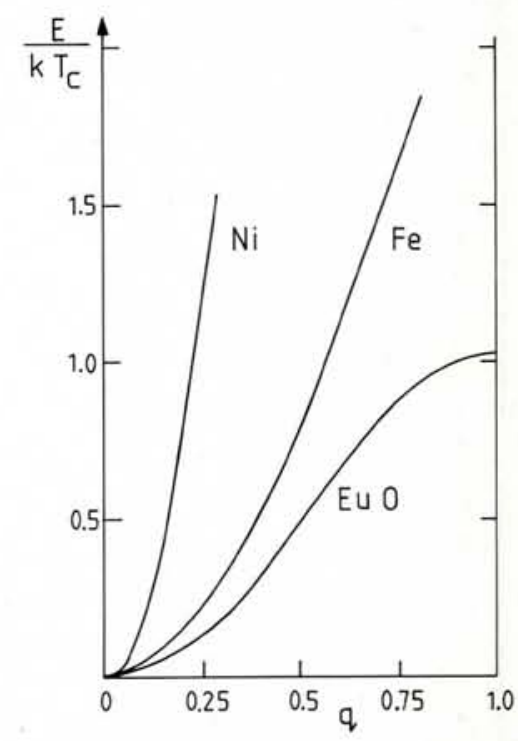

Fig. 2 - The spin wave spectra of iron and nickel in comparison with that of EUO. The energy is given in terms of $E / k T_{c}$ and plotted against the reduced wave-vector $q$. 
Thus the difference between the crosssections for neutrons scattered with reversal of their spin, (spin-flip scattering) when that spin is first parallel to the momentum transfer and then perpendicular to it, has to be purely magnetic in origin; in the paramagnetic regime it is $S(q, \omega) / 2$. Triple axis spectrometers to carry out polarisation analysis experiments have crystal monochromators and analysers which select a particular polarisation state at the same time as a particular range of incident or scattered wave-vectors. Since polarising monochromators and analysers are notoriously inefficient, it is no surprise that the only results to date come from the three laboratories operating high flux reactors - Brookhaven and Oak-Ridge in the USA, and ILL Grenoble France. Even with such reactors some compromises are necessary to ensure a statistically significant signal. At Brookhaven and Oak-Ridge the emphasis has been on high resolution for wave-vectors restricted to the lower third of the Brillouin zone $\left.{ }^{7,8}\right)$. At ILL ${ }^{9,10}$ ) most of the experiments have measured the response in a wide energy range over wave-vectors out to the zone-boundary. Where the results of the different groups overlap, they are in rather good agreement, taking into account the varied experimental conditions, although the methods used for their interpretation have been rather different.

Results of Polarisation Analysis Studies Above $T_{\mathrm{c}}$

The evolution of the paramagnetic scattering above $T_{\mathrm{c}}$ in the range $q<$ $0.25 \AA^{-1}$ follows closely the double Lorentzian form. In the intermediate region $0.25<q<0.5$, whereas the medium resolution results can still be fitted by a double Lorentzian form, those with the best resolution ${ }^{7}$ ) give a fairly sharp peak at finite energy transfer in constant- $q$ scans. This peak gives evidence for the existence of a damped, but still propagating, spin-wave. In iron the critical wave-vector below which the spinwave ceases to propagate is $0.43 \AA^{-1}$ and in nickel it is $0.25 \AA^{-1}$.

Figure 3 shows the Fourier spectrum of the SDSDCF given by $q^{2} \int S(q \omega) d \omega$ at various temperatures above $T_{c}$ up to 1.5 $T_{c}$ for iron and to $2 T_{c}$ for nickel. In all cases, a peak at $q=q_{\max }$ is observed in the intermediate $q$ range indicating that the most frequently occurring configurations in the magnetisation density have approximately ferromagnetic order over a distance of the order $2 \pi / q_{\max }$. For iron this distance (some $15 \AA$ ) is relatively independent of temperature; in nickel it is temperature dependent and varies

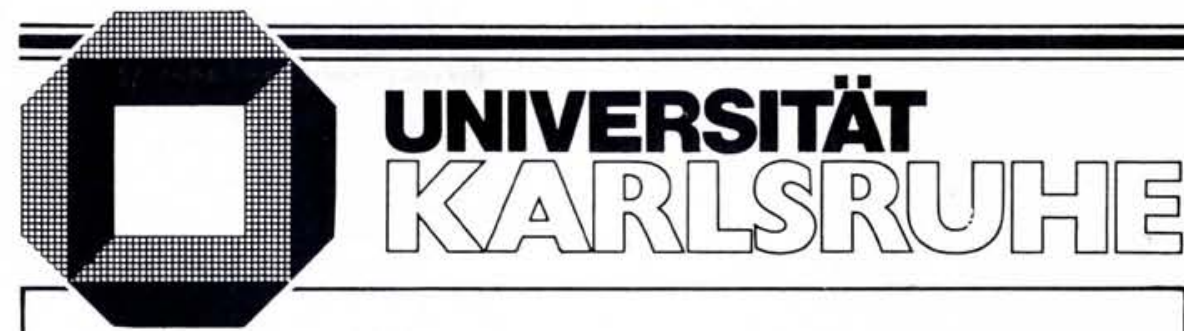

In der Fakultät für Physik ist im Institut für Theorie der Kondensierten Materie die Stelle eines

\section{Professors (C4) für Theoretische Physik}

zu besetzen. Der Bewerber soll sich durch Arbeiten auf dem Gebiet der Physik der Kondensierten Materie ausgezeichnet haben. Es wird erwartet, dass der Stelleninhaber sich an den Lehraufgaben der Fakultät im üblichen Umfang beteiligt.

Die zu besetzende Stelle ist im Rahmen des Förderprogramms (Fiebiger-Plan) für hochqualifizierte jüngere Wissenschaftler neu geschaffen worden. Der Bewerber darf daher das 45. Lebensjahr noch nicht überschritten haben und sollte nicht Inhaber einer C4-Stelle sein.

Bewerbungen mit den üblichen Unterlagen sind bis zum 10. April 1986 zu richten an den Dekan der Fakultät für Physik der Universität Karlsruhe, Postfach 6980, D - 7500 Karlsruhe.

between about $20 \AA$ at $1.2 T_{\mathrm{c}}$ and $12 \AA$ at $2 T_{\text {. }}$.

The scattering observed at the zoneboundary is of some interest, as it is there that a qualitative difference exists between the predictions of the Heisenberg and Stoner theories $\left.{ }^{11}\right)$. In iron this is small and corresponds to about $1.5 \mu_{\mathrm{B}}^{2}$ per atom, no significant scattering being found outside an energy window of 0.6 $k T_{c}$. For nickel the scattering is rather stronger relative to the ordered moment $\left(\simeq 0.7 \mu_{\mathrm{B}}\right)$ and is spread over a much larger range of energies at least up to 3.7 $k T_{\text {c }}$.
Magnetic Structure in the Paramagnetic State

One may ask what picture of the paramagnetic state is presented by all these new data, and what features are special to metallic systems. The data furnish many different indications that the longlived magnetic entities in a paramagnetic metal are regions of correlated magnetisation density, not confined to just a single atom, but extending over several unit cells. The existence of these correlated entities gives rise to the peak in the Fourier spectrum on the SDSDCF. It is quite plausible that spin waves with

\section{$\mathrm{im}^{\mathrm{t}}$ INSTITUT DE MICROTECHNIQUE DE L'UNIVERSITÉ Rue de la Maladière 71 CH - 2000 NEUCHĀTEL}

\section{Pour des postes de chefs de travaux (Oberassistent)}

\section{nous cherchons 2 PHYSICIENS (post-doc)}

\section{DOMAINE OPTIQUE APPLIQUÉE (Prof. R. Dändliker)}

- Préparation et gestion des travaux pratiques, travaux de semestre et de diplôme.

- Recherche dans le domaine du traitement de l'information par voie optique (optical computing) ou dans le domaine des fibres optiques (capteurs à fibres optiques).

DOMAINE CAPTEURS MINIATURISÉS EN SILICIUM (Prof. N. de Rooij)

- Préparation et gestion des travaux pratiques, travaux de semestre et de diplôme.

- Recherche dans le domaine de la conception, réalisation et caractérisation des capteurs miniaturisés en silicium pour mesurer des grandeurs physiques (pression, température) et chimiques (concentration d'ions ou de gaz).

Entrée en fonction: 1er avril 1986
Tél. (38) 246000 
wave lengths less than the characteristic dimension $2 \pi / q_{\max }$ can propagate within the correlated entities and one may note the correspondence between $q_{\max }$ and the critical cut-off wave-vector for the spin waves. It appears that there are two different energy scales which are important. The first is that of the Fermi energy and the exchange splitting of the d-band, expected to be of the order of electron volts and to be less for nickel than for ion. This exchange splitting leads to atomic-like moments, but because of the itinerant nature of the electrons, the magnetisation cannot be localised on a single atom. The second energy scale is that of the coupling between the magnetic entities and is of order $k T_{c^{\prime}}$ so that the ferromagnetic/paramagnetic transition at $T_{c}$ is driven by transverse fluctuations leading to disorder of the magnetic entities.

This picture of the paramagnetic state is consistent with the magnetic excitations in the ferromagnetic state. Indeed the spin wave measurements show that only the low $q$ spin-waves renormalise to zero at $T_{c^{\prime}}$ indicating that only longrange correlation is lost in the paramagnetic transition. The energies of spinwaves with q $>0.25 \AA^{-1}$ only fall a little on approaching $T_{c}$ thermal energies, being still insufficient to create excitations with wave-vectors greater than about one third of the zone boundary value. These data therefore also suggest that the magnetic entities which disorder at $T_{\mathrm{c}}$ are not confined to single atoms, but that reversal of an electron spin at one site is followed on a short time-scale by reversal of a surrounding cloud of magnetisation involving many neighbouring atoms.

The internal dynamics of these magnetic entities are the subject of continuing studies, both experimental and theoretical. At present the experimental evidence suggests that in iron the coupling energy within the entities is very large compared with $k T_{c}$. This explains why, in the ordered state, spin waves continue to be the dominant excitation even up to $1.8 k T_{c^{\prime}}$ and in the paramagnetic state the correlation length is largely independent of temperature up to $1.5 k T_{c}$. Additionally in the paramagnetic state, scattering at the zoneboundary which would come from fluctuations in the magnitude or direction of the local (one-site) magnetisation, are not observed up to more than twice $k T_{\text {c }}$. In nickel the difference in the two energies is probably much smaller. The spin waves are observed to enter the Stoner continuum at around $1.5 k T_{c}$. In the paramagnetic state the correlation
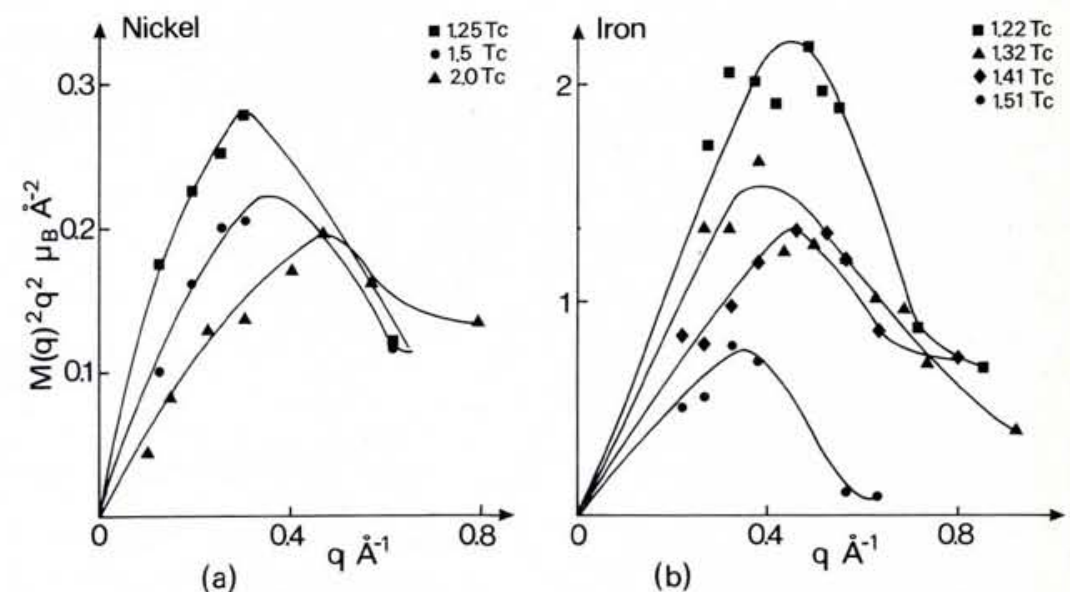

Fig. 3 - The Fourier spectrum of the SDSDCF for nickel and iron at various temperatures above $T_{c}$. The full lines have been inserted simply as a guide to the eye.

length is temperature dependent in the range $k T=(1.3-2) k T_{c}$ and scattering at the zone boundary is found in a wide energy range with a maximum around $1.85 k T_{c}$.

\section{Recent Theoretical Developments}

There are currently three essentially different theoretical approaches which attempt to describe the finite temperature properties of metallic ferromagnets. The first ${ }^{11,12,13}$ ) starts from a mean field theory and introduces paramagnetic disorder using the random phase approximation; short range order above the Curie temperature comes from interaction between local moments via the conduction band. At the present stage of development these theories seem unable to account for the full range of finite temperature properties with a single set of parameters. An alternative type of model ${ }^{14.15}$ ) starts from many-body theory with the introduction of local magnetic coordinates to describe the fluctuations above $T_{c}$. Local order above $T_{c}$ is due to phase coherence of electronic wave-functions over distances considerably larger than the interatomic spacing. These fluctuating band theories seem capable of giving a coherent account of both the structure above $T_{\text {c }}$ and the magnetic excitations. Finally Moriya ${ }^{16}$ ) has developed a theory which allows a unified treatment of both local moment and itinerant systems by considering interaction between different modes of spin fluctuations. He introduces a new collective mode of longitudinal spin fluctuations which may be important for understanding the excitations near the zone-boundary in nickel.

\section{Conclusion}

In recent years new insight into the magnetic state of metallic ferromagnets in the paramagnetic state has been obtained, mainly as a result of neutron scattering studies. The results of these studies show that metallic magnets, in which magnetic electrons take part in the Fermi surface, owe their characteristic properties to a magnetic coupling energy large compared with $k T_{c}$ and having a range of several interatomic distances. The observations have encouraged theorists to develop new models to describe itinerant magnetism at finite temperatures. Out of these developments has come, not only a better understanding of the $3 d$ ferromagnetic metals themselves, but also new insight into the properties of other magnetic systems such as very weak metallic magnets and heavy fermion systems.

\section{REFERENCES}

1. Stoner E.C., Journal de Physique 12 (1951) 372.

2. Izuyama T., Kim D. and Kubo R., J. Phys. Soc. Japan 18 (1963) 1025

3. Marshall W. and Lovesey S.W., Theory of Thermal Neutron Scattering (O.U.P.) 1961.

4. Lynn J.W., Phys. Rev. B 11 (1975) 2624.

5. Lynn J.W. and Mook H.A., Phys. Rev. B 23 (1981) 198.

6. Loong C.-K., Carpenter J.M., Lynn J.W., Robertson R.A. and Mook H.A., J. Appl. Phys. 55 (1984) 2624.

7. Mook H.A. and Lynn J.W., J. Appl. Phys. 57 (1985) 3006.

8. Shirane G., Steinsvoll O., Uemura Y.J. and Wicksted J., J. Appl. Phys. 55 (1984) 1887. 9. Brown P.J., Ziebeck K.R.A., Deportes J. and Givord D., J. Appl. Phys. 55 (1984) 1881. 10. Brown P.J., Capellmann H., Deportes J., Givord D., Johnson S.M., Lynn J.W. and Ziebeck K.R.A., J. Physique 46 (1985) 827. 11. Herring C., Magnetism IV, Ed. G.T. Rado and H. Suhl (1962) 132.

12. Shastry B.S., Edwards D.M. and Young A.P., J. Phys. C 14 (1981) L665.

13. Hasegawa H., J. Phys. Soc. Japan 49 (1980) 963.

14. Prange R.E. and Korenmann V., Phys. Rev. B 19 (1979) 4691.

15. Capellmann H., Z. Phys. B 34 (1979) 29.

16. Moriya T., J. Mag. Mag. Mat. 31-34 (1983) 10. 